\title{
Diagnosis and treatment of abnormal dental pain
}

\author{
Ken-ichi Fukuda \\ Division of Special Needs Dentistry and Orofacial Pain, Department of Oral Health and Clinical Science, Tokyo Dental College, Tokyo, \\ Japan
}

\begin{abstract}
Most dental pain is caused by an organic problem such as dental caries, periodontitis, pulpitis, or trauma. Diagnosis and treatment of these symptoms are relatively straightforward. However, patients often also complain of abnormal dental pain that has a non-dental origin, whose diagnosis is challenging. Such abnormal dental pain can be categorized on the basis of its cause as referred pain, neuromodulatory pain, and neuropathic pain. When it is difficult to diagnose a patient's dental pain, these potential alternate causes should be considered. In this clinical review, we have presented a case of referred pain from the digastric muscle (Patient 1), of pulpectomized (Patient 2), and of pulpectomized pain (Patient 3) to illustrate referred, neuromodulatory, and neuropathic pain, respectively. The Patient 1 was advised muscle stretching and gentle massage of the trigger points, as well as pain relief using a nonsteroidal anti-inflammatory and the tricyclic antidepressant amitriptyline. The pain in Patient 2 was relieved completely by the tricyclic antidepressant amitriptyline. In Patient 3, the pain was controlled using either a continuous drip infusion of adenosine triphosphate or intravenous $\mathrm{Mg} 2+$ and lidocaine administered every 2 weeks. In each case of abnormal dental pain, the patient's diagnostic chart was used (Fig.2 and 3). Pain was satisfactorily relieved in all cases.
\end{abstract}

Key Words: Dental pain; Referred pain; Neuromodulation; Neuropathic pain.

\section{INTRODUCTION}

When a patient visits our clinic complaining of dental pain, we diagnose the cause by looking-both visually and radiographically - for organic or functional abnormalities. Nearly all pain is caused by an organic problem such as dental caries, periodontitis, pulpitis, or trauma. Diagnosing the cause of these symptoms is relatively straightforward, and by eliminating the source of the problem, we can readily eliminate the pain. However, patients often also complain of dental pain that has a non-dental origin; diagnosing the cause of such pain is challenging. In this article, I describe this type of pain and discuss how to manage such complaints using examples from individual cases.

\section{CASE REPORT}

Patient 1: A 28 year-old woman presented with the following complaint: "My lower central incisors hurt." Upon examination, no apparent caries or periodontitis was found. X-rays were taken, but there were no tooth fractures or other abnormalities (Fig. 1). Furthermore, no occlusal or percussion pain was present.

The periapical gingivae of the mandibular central incisors were anesthetized using infiltration anesthesia, but the pain did not resolve. Let us consider categories and the referred pain and neuromodulation (Fig. 2). The patient was not experiencing a throbbing pain, and none of the symptoms were present that are usually seen with cluster headaches. Moreover, she did not give the impression of having depressive tendencies. However,
Copyright(C) 2016 Journal of Dental Anesthesia and Pain Medicine
Received: 2016. March. 20. • Revised: 2016. March. 30. Accepted: 2016. March. 31. Corresponding Author: Ken-ichi Fukuda, Division of Special Needs Dentistry and Orofacial Pain, Department of Oral Health and Clinical Science, Tokyo Dental College, 2-9-18 Misakicho, chiyoda-ku, Tokyo, 101-0061, Japan Tel: +81-3-6380-9243 Fax: +81-3-5275-1750 E-mail: kfukuda@tdc.ac.jp 
she did say, "Recently, work has been busy, and maybe the psychological stress has been piling up." We investigated the digastric muscle externally, and then from within the oral cavity. When a certain point was pressed, the patient said, "I can feel it in my tooth! That's where it hurts!". Thus, a trigger point for sensation in the mandibular central incisors was discovered; the same phenomenon was then discovered by probing within the oral cavity. Referred pain from the digastric muscle was then diagnosed.
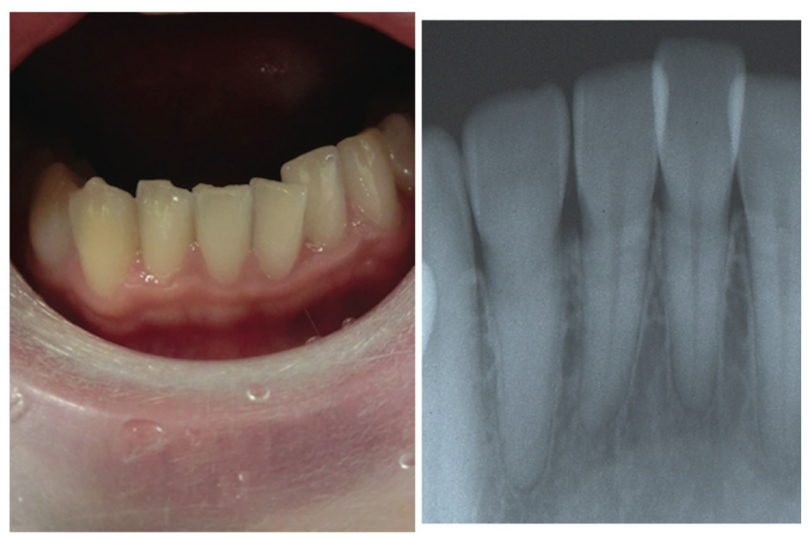

Fig. 1. A 28 year-old female with the complaint, "My lower central incisors hurt".
This condition should be treated using muscle stretching techniques that extend and rotate the neck, as well as gentle massage of the trigger points. A non-steroidal anti-inflammatory should also be prescribed-because the condition involves inflammation caused by muscle fatigue, these drugs are sometimes effective. If several days pass and the pain has still not subsided, antiinflammatory treatment is considered ineffective. The tricyclic antidepressant amitriptyline reinforces the inhibition of descending pain, thus applying a brake to pain transmission routes; the drug also controls pain acceleration. It mediates analgesia via several mechanismsnamely, sodium channel blocking and operation on opioid receptors - and it is very effective in treating referred pain from muscles. However, the drug does have adverse effects, including dizziness, oral drying, and palpitations; these should be fully explained to the patient before prescribing. The starting dosage is $10 \mathrm{mg} /$ day, and this is increased gradually while the propitious and adverse effects are observed.

Referred pain originating in the muscles, as was seen in this case, is surprisingly common. Upper molar pain

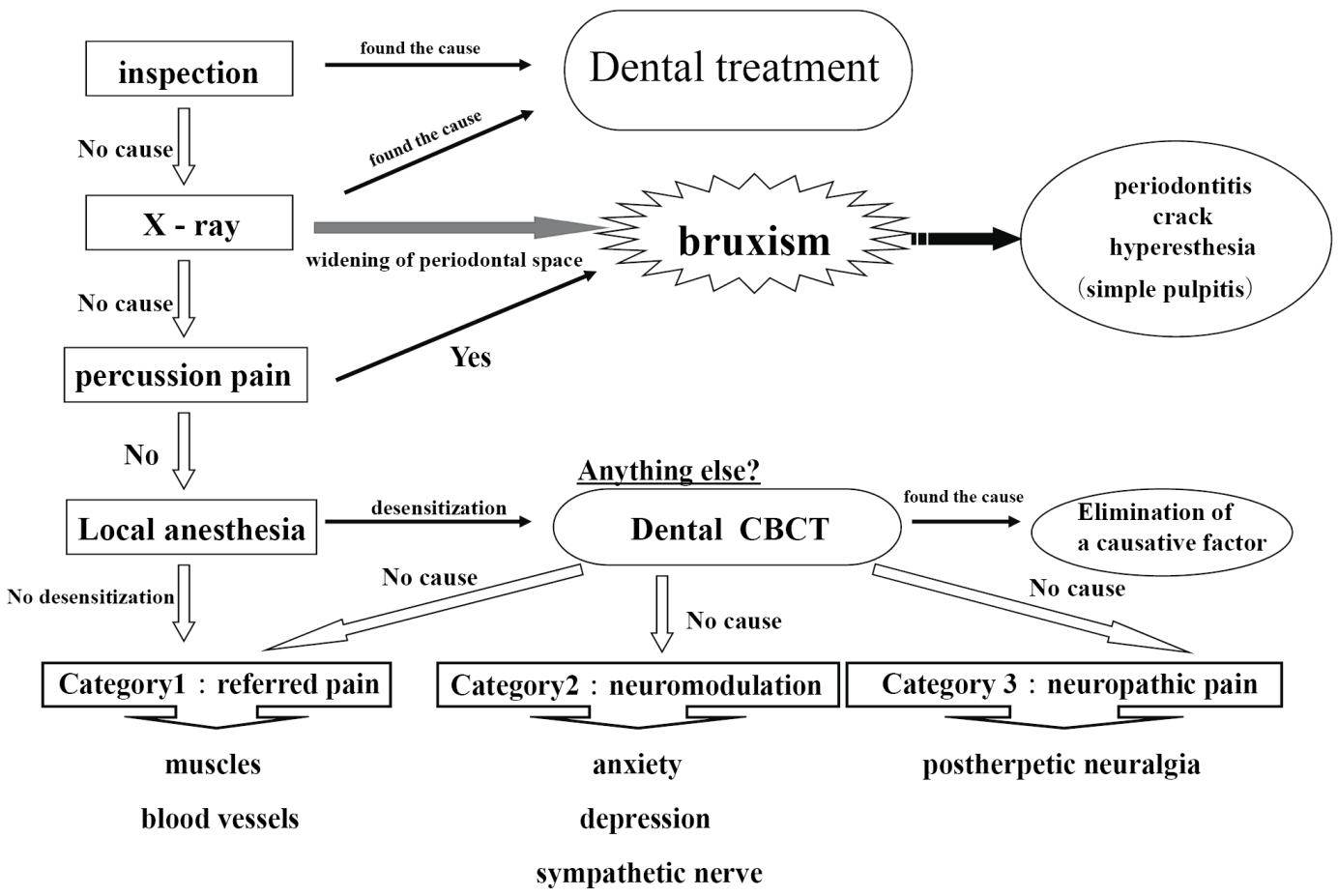

Fig. 2. Diagnostic chart for abnormal dental pain. 
referred from the temporal muscles, as well as lower molar pain referred from the masseters, is particularly frequent. A local anesthetic, or saline, can be injected at the trigger point to reach the temporal muscles or masseters, but it is difficult to apply this technique in the case of the digastric muscle. Other treatments include acupuncture, near-infrared therapy, centrally acting muscle relaxants, and splint therapy.

Patient 2: A 45 year-old woman was complaining of dental pain. Pain was continuous, deep, throbbing. After the left maxillary second premolar was pulpectomized, the pain had not abated. Neither did the pain resolve after a number of successive root canal treatments. Indeed, after the pulpectomy, 6 months passed before the root canal procedure; the pain persisted throughout. No problems had occurred with the cotton plug inserted into the root canal, and there was no percussion pain or pain on reamer insertion. A field-block anesthesia was carried out in the left first premolar, and a local anesthetic was injected into the root canal, but the effect was insufficient. Let us consider the referred pain and neuromodulation (Fig. 3) - the patient was not experiencing throbbing pain, and none of the symptoms associated with cluster headache were seen. She was somewhat exhausted because of the ongoing pain, and the results of psychological testing on the Hospital Anxiety and Depression Scale were false-positive for depression. There were no trigger points in the masticatory muscles. Category 2 neuromodulation was diagnosed (Fig. 3).

This condition can be treated using amitriptyline. In this case, the starting dosage of the drug was $10 \mathrm{mg}$ /day; this was increased to $60 \mathrm{mg} /$ day after 40 days. At that point, the pain had been completely controlled. Fifty-three days after the initial examination, the root canal was filled without incident (Fig. 4). The prescription was continued at the same dosage for a further 10 months, and then decreased gradually. After 1 year and 2 months, the prescription was discontinued, and there was no residual pain. Conditions in which pain persists long after pulpectomy used to be called "atypical odontalgia", and tricyclic antidepressants were highly effective in treating such cases. Therefore, this case was a classic one.

Patient 3: A 39 year-old woman was complaining of dental pain. After pulpectomy of the left mandibular second molar, the pain had not abated; neither had it resolved after a number of successive root canal

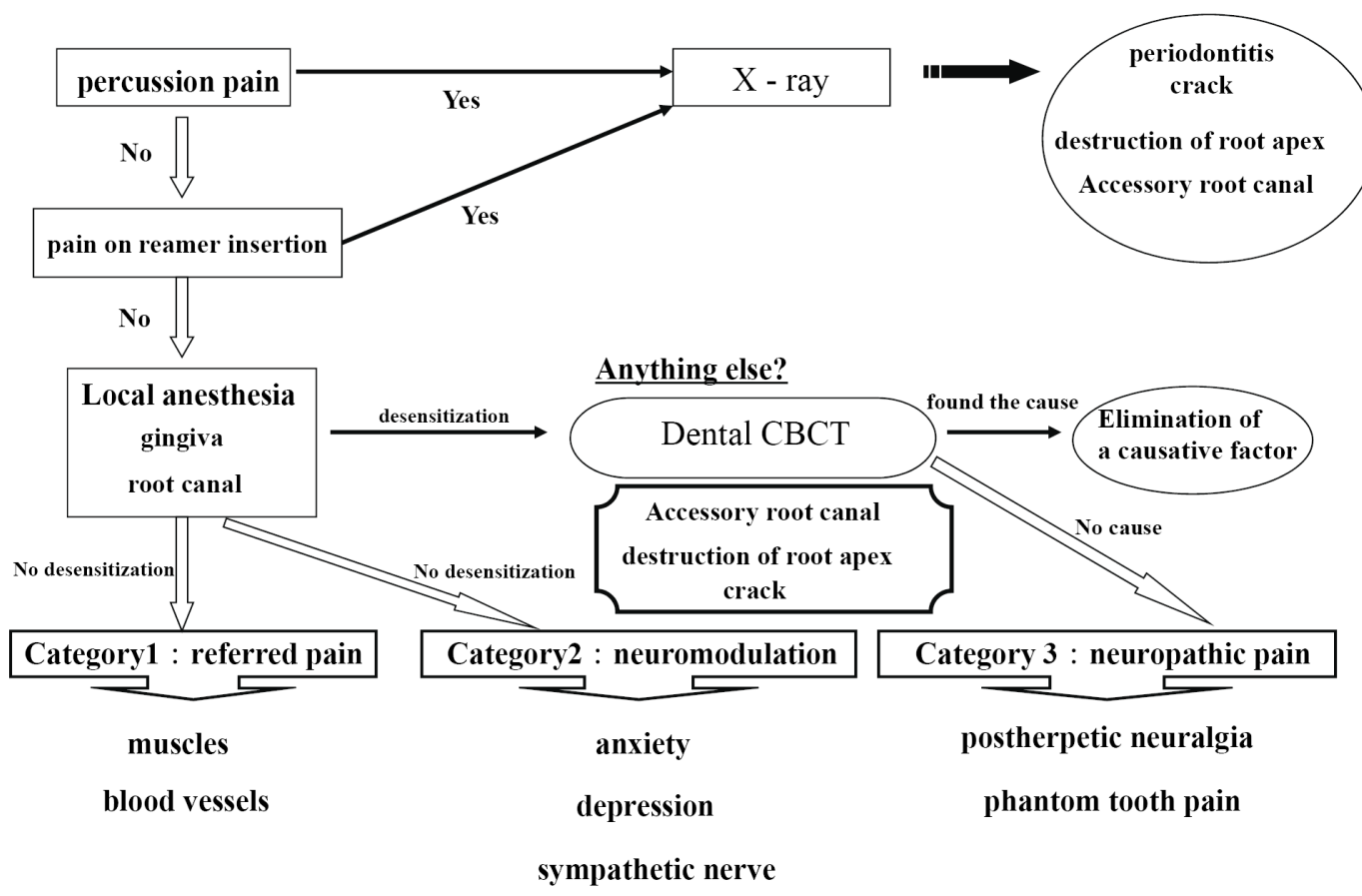

Fig. 3. Diagnostic chart for abnormal pulpectomized dental pain. 


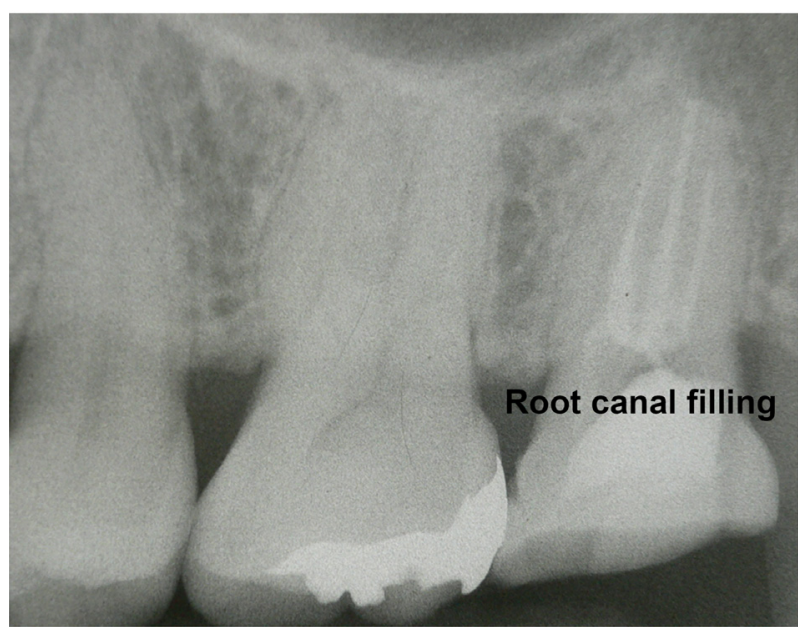

Fig. 4. The root canal was filled without incident.

treatments. Indeed, the root canal therapy caused more intense pain. Throughout the 5 months after pulpectomy, the pain had persisted stubbornly and caused some sleepless nights. No problems had occurred with the cotton plug inserted in the root canal; there was no percussion pain, and reamer insertion was painful occasionally. The left mandibular second molar field was blocked, and a local anesthetic was injected into the root canal; pain was relieved in a very short time. On dental cone beam CT and microscopy, there were no clear anomalies. Allodynia and desesthesia were observed on the mucosal surfaces around the tooth. Therefore, let us consider category 3 (neuropathic pain; Fig. 3).

This was a case of phantom tooth pain. Carbamazepine (600 mg/day) and amitriptyline (90 mg/day) decreased pain slowly, but were insufficient. Other tricyclic antidepressants were also ineffective, as were the antiepileptic drug clonazepam, and mexiletine hydrochloride. Intravenous ketamine, $\mathrm{Mg}^{2+}$, and/or lidocaine provided temporary, dramatic relief from pain. Continuous drip infusion of adenosine triphosphate (ATP) sustained a pain-relieving effect for approximately 2 weeks, so the patient was prescribed continuous drip infusion of ATP and introvenous lidocaine every 2 weeks. ATP is immediately decomposed in the blood into adenosine; intravenous administration of drugs causes an analgesic effect that is mediated by A1 receptors. Continuous drip

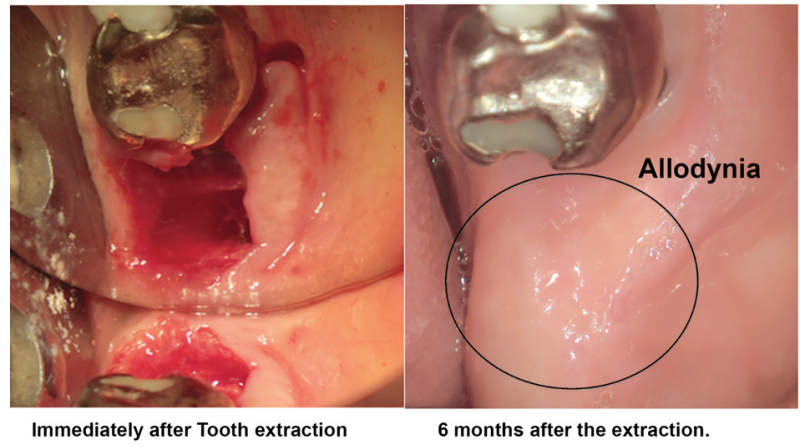

Fig. 5. Allodynia was obsereved on mucosal surfaces following the extraction.

infusion, for 2 hours or longer, at a rate of $5-6 \mathrm{mg} / \mathrm{kg} / \mathrm{h}$ can provide temporary, dramatic relief of phantom tooth pain [1]. The effect is slow to begin, but its duration can be anywhere from several days to several weeks. Phantom tooth pain cannot be relieved, even by tooth extraction. In this case, the tooth was extracted ten years later because of fracture, and the symptoms remained unchanged. Allodynia was observed on the mucosal surfaces following the extraction (Fig. 5). The patient is still given a continuous drip infusion of ATP, or intravenous $\mathrm{Mg}^{2+}$ and lidocaine, every 2 weeks to control her pain. She is also treated using oral stent therapy with capsaicin cream.

\section{DISCUSSION}

What kind of pain is difficult to diagnose? First, let us consider the mechanism that mediates pain. Pain is a sensory function that informs our brain when infection or trauma has disrupted a tissue in the body, or when a part of the body has become fatigued. Malfunctions in this mechanism can threaten life in various ways. Fig. 6 shows a patient with congenital insensitivity to pain combined with anhidrosis; because the patient feels no pain, the entire body is covered with wounds.

Pain, as we can see, warns us of a problem in the human body, and it is therefore an essential defense mechanism that ensures a healthy life. Where teeth are 


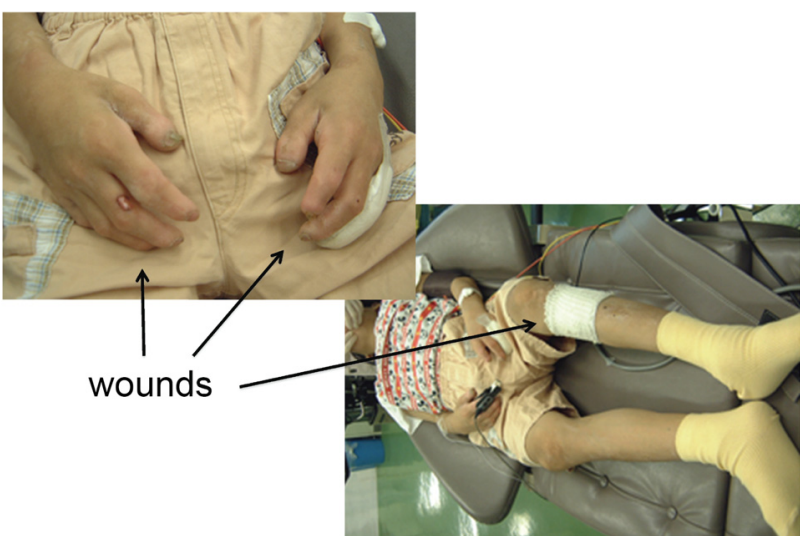

Fig. 6. A patient with congenital insensitivity to pain with anhidrosis

concerned, pain is the alarm that warns us of threatening situations, such as invasion by cariogenic bacteria, or dental fracture. When this alarm is working properly, there are no problems. However, the transmission mechanisms for pain are complex; sometimes they indicate pain in a way that confounds the dentist. Pain signals originating in peripheral sensory nerve receptors travel through innumerable neurons to the somatosensory cortex, where the signal is recognized as pain. Along the transmission route, various modifications are made by a variety of neural networks, such as the descending pain inhibitory system, which acts like a brake on transmission, the excitatory system, which plays the role of accelerator, and the emotional or sympathetic nervous system (Fig. 7). For example, if for some reason the brake (the inhibitory system) fails or the accelerator (the excitatory system) over-functions, information is transmitted and produces pain that would under normal circumstances not be perceived. Such pain confounds diagnosis.

But what type of pain is this? Why does this sort of pain, or abnormal dental pain for that matter, occur? Dental pain that frustrates diagnosis can be categorized on the basis of its cause: (1) pain that is perceived at a location different from its source, (2) pain that results from neuromodulation, or (3) neuropathic pain. When it is difficult to diagnose a patient's complaint of dental pain, these potential alternate causes should be kept in mind.

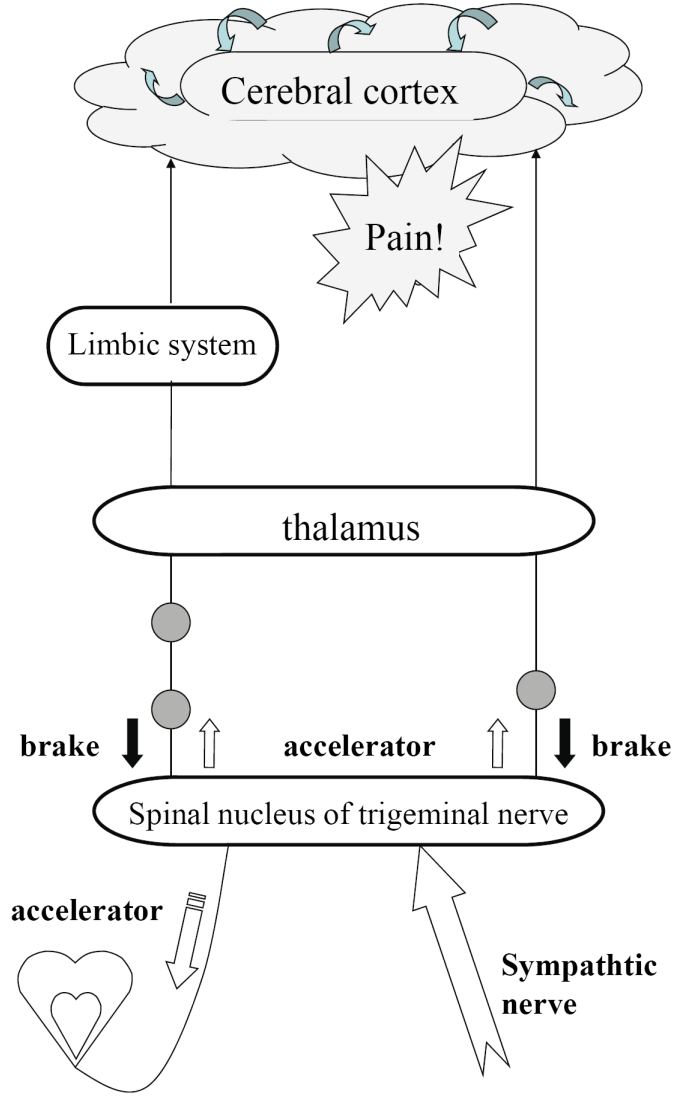

Fig. 7. Mechanism that produces pain.

\section{Category 1: pain perceived at a location different from its source}

Pain that occurs at a site separate from its origin is called "referred pain." Even if the origin of the pain is a location other than the teeth, dental pain can occur, and the patient will complain of a toothache. However, the cause of the dental pain is hidden, confounding diagnosis by a dentist. So then, what type of mechanism produces referred pain? Information transmitted not only from teeth but also from various other sites such as the muscles and blood vessels, is bundled together at the synapses of trigeminal nerve neurons - both primary and second-order -as well as at the spinal nucleus of the trigeminal nerve (Fig. 8). For example, pain in the masseter muscles is transmitted through the masseteric nerve before reaching these points. The inferior alveolar nerve also transmits to these points - from the molar region of the lower jaw. When masseteric pain persists, the pain-transmitting 


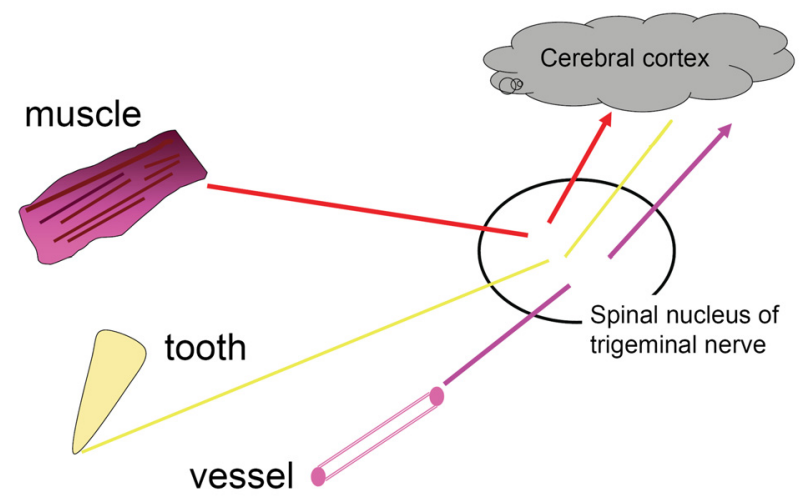

Fig. 8. Information transmitted from various sites is bundled together at the synapses of trigeminal nerve.

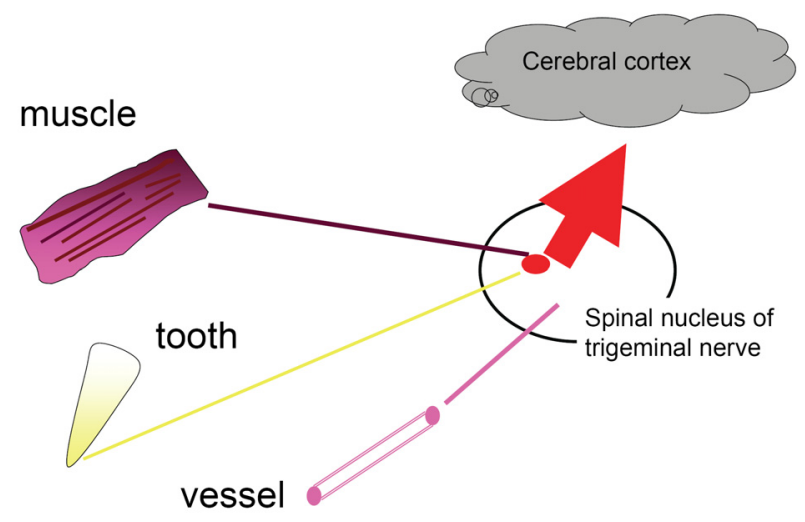

Fig. 9. Referred Pain.

\section{Trigger points of masseter muscle}

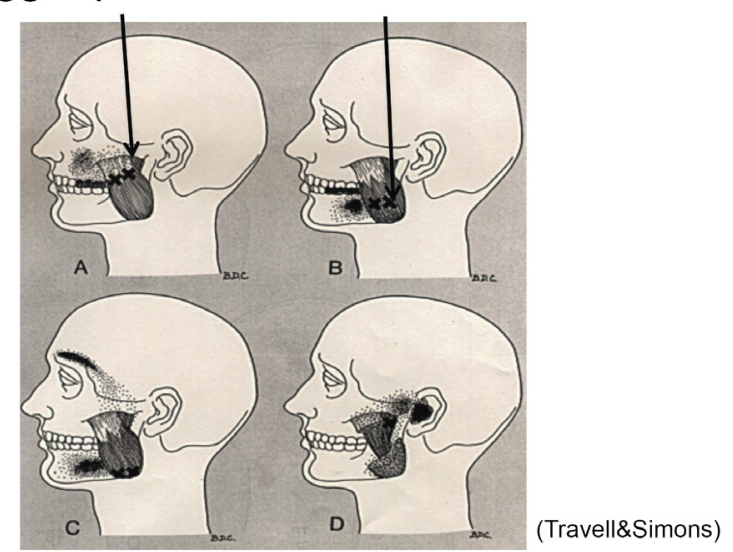

Fig. 10. Trigger point of masseter muscle.

neurons become hypersensitive, a phenomenon called central sensitization; in this way, the accelerator of pain transmission is figuratively pressed down. Subsequently, the slightest stimulus propagates to the central nerves via

\section{Trigger points of temporal muscle}

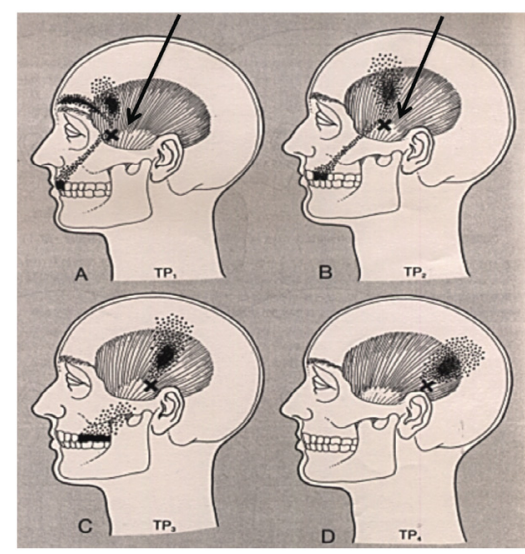

(Travell\&Simons)

Fig. 11. Trigger point of temporal muscle.

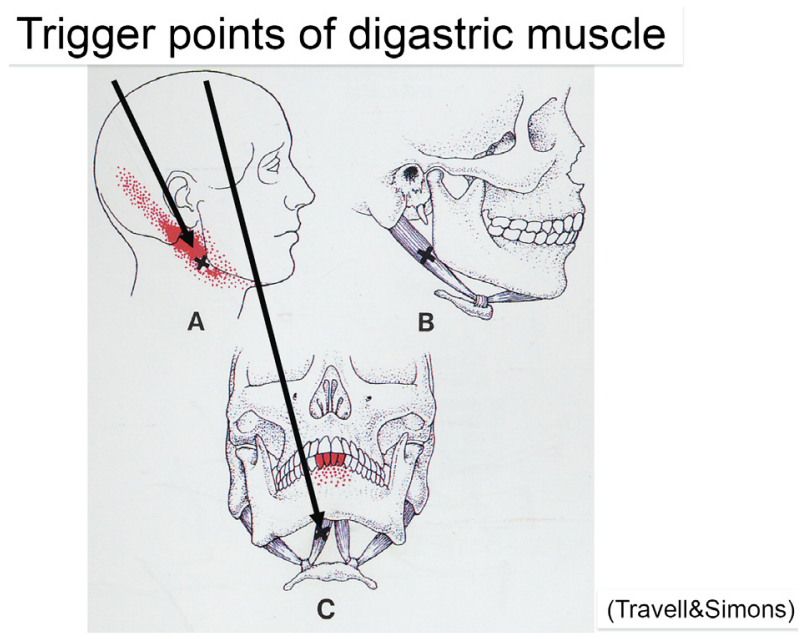

Fig. 12. Trigger point of digastric muscle.

neurotransmission. As a result, pain that originally developed in the masseters is felt as pain traveling through the inferior alveolar nerve, i.e., mandibular molar pain (Fig. 9). The source of pain at a specific site in the masseter (shown by the $\mathrm{X}$ ), produces a referred pain at another location, namely, the mandibular molar region (shown in black) (Fig. 10) [2]. Similarly, a source of pain in the temporal muscle produces a referred pain in the maxillary molar region (Fig. 11) [2], and a source in the digastric muscle produces a referred pain at the mandibular central incisors (Fig. 12) [2]. These types of dental pain are termed muscular toothaches. Consequently, the pain is not relieved, even if the teeth are treated. When sites known as trigger points (indicated by the $\mathrm{X}$ ) 
are pressed, the patient perceives a sensation in the black region. When the attending dentist notes such perception, the condition can be promptly diagnosed.

Nonetheless, referred pain is not associated with muscles alone. Migraine headaches, cluster headaches, and other neurovascular headaches can also be referred to the teeth; in such cases, they are termed neurovascular toothaches. In neurovascular headaches, neuropeptides released from trigeminal nerve endings in the intracranial blood vessels stimulate and dilate the blood vessels; the consequent inflammation is thought to produce pain. The same mechanism produces dental pain. Referred dental pain from migraine headaches causes episodic, pulsating vascular pain - often in the upper and lower premolars, as well as in the upper canines. If accompanied by a headache, diagnosis is relatively straightforward; first of all, patients do not normally visit the dentist for headache treatment. However, examination and diagnosis are more complicated in cases that present as toothache alone-the subjective symptoms of pulpitis are similar, and practitioners must take care to avoid inappropriate pulpectomy.

Finally, pain from internal organs can cause a referred dental pain known as visceral toothache. Visceral toothache involves referred pain from the heart or stomach. Cardiac toothache occurs when pain from angina pectoris is referred to the teeth; toothache may also precede the onset of heart disease.

\section{Category 2: pain from neuromodulation}

The transmission of pain stimuli can be modulated by accumulated psychological stress or abrupt changes in emotions. In the cerebrum - the final stop on the transmission route-information from various sources is integrated and processed to complete the perception of what we term pain; psychological states and prolonged pain are thought to have a major effect here. The sympathetic nervous system can also affect the transmission of pain stimuli; when it does, severely debilitating pain can develop. Such is the case in nearly all patients with orofacial pain or toothache of unknown cause who are transferred from emergency facilities as outpatients. Their pain can be relieved dramatically by intravenously injecting antianxiety agents, or by stellate ganglion block.

\section{Category 3: neuropathic pain}

The nervous mechanisms that transmit pain stimuli are extremely complex. For this very reason, when a disorder arises in one part of a transmission route, the complexity increases; consequently, an abnormal sensation or pain stimulus different from the original may be transmitted. For example, in many cases, once the trunk of the inferior alveolar nerve is injured, complete recovery is unlikely, because the trunk comprises nerves that are responsible for pressure sensation, tactile sense, sense of temperature, and cold sensation, as well as for algesthesia. In the recovery process following injury, these routes simply get jumbled [3]. Nevertheless, nearly all fine-pulp nerves branching from the inferior alveolar nerve are made up of algesic fibers; in many cases, no transmission abnormalities persist as sequelae, even if the nerves are severed. For this reason, nerve-severing treatments for pulpectomy or tooth extraction used to be performed in ordinary dental clinics, in the belief that neuropathic pain would not occur, even if pulp nerves were severed. However, it is easy to imagine abnormal pain developing after pulpectomy or tooth extraction. The term "phantom tooth pain" has been used for some time to describe symptoms akin to the neuropathic "phantom limb pain" that follows a digital amputation [4]. This condition can cause pain symptoms that frustrate diagnosis after pulpectomy or tooth extraction.

In conclusion, I have presented the cases of three patients with abnormal dental pain. They were each suffering from a respective type of pain-referred pain, neuromodulation, and neuropathic pain. The diagnostic chart was used in each case. All patients were satisfied with pain relief. Pain is burdensome for anyone; the mission of the doctor is to provide relief as quickly as possible for patients complaining of facial or dental pain. Correct diagnosis is crucial in this regard. 


\section{REFERENCES}

1. Fukuda K, Hayashida M, Fukunaga A, Kasahara M, Ichinohe T, Kaneko Y. Pain-relieving effects of intravenous adenosine 5 '-triphosphate (ATP) in chronic intractable orofacial pain: an open-label study. J Anesth 2006; 21: 244-50.

2. Travell JG, Simons DG. Myofascial Pain and Disfunction:
The Trigger Point Manual. 1st ed. Baltimore, Williams and Wilkins. 1983.

3. Fukuda K, Ichinohe T, Kaneko Y. Pain management for nerve injury following dental implant surgery at Tokyo Dental College Dental College Hospital. Int J Dent 2012; Article ID 209474, 9 pages.

4. Marbach JJ, Hulbrock J, Hohn C, Segal AG. Incidence of phantom tooth pain: an atypical facial neuralgia. Oral Surg Oral Med Oral Pathol 1982; 53: 190-3. 